\title{
Collaboration in pharmacovigilance: lamotrigine and fatal severe cutaneous adverse reactions - a review of spontaneous reports
}

This article was published in the following Dove Press journal:

Therapeutics and Clinical Risk Management

20 July 2017

Number of times this article has been viewed

\author{
Neil Brickel' \\ Haris Shaikh' \\ Andrew Kirkham ${ }^{2}$ \\ Greg Davies' \\ Michelle Chalker ${ }^{\prime}$ \\ Pascal Yoshida ${ }^{3}$ \\ 'Global Clinical Safety and \\ Pharmacovigilance, GlaxoSmithKline, \\ Uxbridge, Middlesex, UK; ${ }^{2}$ Classic \\ and Established Products, \\ GlaxoSmithKline, Brentford, \\ Middlesex, UK; ${ }^{3}$ Clinical Safety \\ and Post-marketing Surveillance, \\ GlaxoSmithKline KK, Tokyo, Japan
}

Correspondence: Neil Brickel Global Clinical Safety and Pharmacovigilance, GlaxoSmithKline, I-3 Iron Bridge Road, Stockley Park, Uxbridge, Middlesex UBI I IBT, UK

Tel +442089902582

Email neil.r.brickel@gsk.com

\begin{abstract}
Pharmacovigilance presents many challenges, particularly when managing unpredictable, rare conditions, eg, severe cutaneous adverse reactions (SCARs). Such rare events are often only detected from spontaneous reports, which present their own limitations, particularly during a prolonged global launch schedule. GlaxoSmithKline's routine pharmacovigilance includes regular reviews of global adverse event (AE) reports and aggregate data from a central safety database. Lamotrigine is one of the several antiepileptic drugs associated with SCARs. After identification of increased rates of fatal SCAR cases with lamotrigine in Japan between September and December 2014, this analysis investigated the global incidence of fatal SCARs with lamotrigine and explored whether known risk factors may have contributed to these cases. Global fatal SCAR cases reported with lamotrigine administration from launch until January 2015 were reviewed for evidence of temporal association with dosing and the presence of risk factors, including comorbidities, concomitant medications, and noncompliance with the prescribing information (PI). Worldwide, the estimated cumulative exposure to lamotrigine was $>8.4$ million patient-years. Globally, there were 54,513 AE reports for lamotrigine, of which 3,454 (6.3\%) concerned SCARs. Of these, $122(3.5 \%)$ had a fatal outcome (attributable and nonattributable to lamotrigine), equating to 0.01 fatal SCARs per 1,000 patient-years. In Japan (estimated cumulative exposure 141,000 patient-years), 17 fatal SCARs were reported (attributable and nonattributable), equating to 0.12 per 1,000 patient-years. Seventy-one percent of fatal SCAR cases in Japan showed evidence of noncompliance with the recommended dosing regimen; in $65 \%$ of the cases, a delay in discontinuation of lamotrigine after early signs of hypersensitivity was reported. Despite a number of limitations inherent in comparing spontaneous report data, this analysis highlights the need for adherence to the lamotrigine PI and emphasizes the importance of collaboration between global and local pharmacovigilance departments, to promptly identify and reduce the risk of rare and serious events, such as SCARs.
\end{abstract}

Keywords: antiepileptic drug, pharmacovigilance, Stevens-Johnson syndrome, toxic epidermal necrolysis, drug reaction with eosinophilia and systemic symptoms

\section{Introduction}

There are many ongoing challenges for pharmacovigilance systems that manage drug safety surveillance across the world. Five principal interrelated activities have been identified as critical in order to address these challenges. These are engaging the public, collaboration and partnerships, the incorporation of informatics, the adoption of a global approach to pharmacovigilance, and evaluation of the impact of pharmacovigilance activities. ${ }^{1}$ This short report illustrates the importance of these activities from the perspective of a pharmaceutical company, GlaxoSmithKline (GSK), dedicated 
to ensuring the safety of patients using its medicines. While the significance of collaborations between pharmaceutical companies, academia, regulatory agencies, and specialist monitoring centers is well recognized, in this report, we highlight the importance of internal collaboration between global and local safety departments as part of a global approach to pharmacovigilance. Such an approach is complicated by wide regional variation in the quantity and quality of spontaneous reporting of adverse events (AEs), even for those as serious as severe cutaneous adverse reactions (SCARs). ${ }^{2}$ In addition to the regional variation in AE reporting, one of the fundamental challenges of evaluating safety based on spontaneous reporting is the lack of reliable exposure data. Unlike clinical trials, or even many observational studies, there are no accurate denominator data for spontaneous reports. The best that can be achieved globally are estimates based on sales volume data, which are accepted by regulatory agencies for periodic reporting purposes as acknowledged in the industry guidance on Good Pharmacovigilance Practices and Pharmacoepidemiologic Assessment issued by the US Food and Drug Administration. ${ }^{3}$ However, despite the limitations inherent in the reporting of spontaneous cases, spontaneous AE reports remain at the core of pharmacovigilance because they help to identify or further characterize AEs, once a medicine is used in a real-world setting. ${ }^{4}$ This is particularly true for rare, unpredictable events such as SCARs. ${ }^{4}$ The example presented in this article with lamotrigine also seeks to highlight the potential challenges associated with the global launch of a drug over a prolonged period of time, particularly when a major country such as Japan is one of the later countries to introduce the medicine to its market.

SCARs include a number of conditions such as Stevens-Johnson syndrome (SJS), toxic epidermal necrolysis (TEN), erythema multiforme, and drug reaction with eosinophilia and systemic symptoms (DRESS, also known as druginduced hypersensitivity syndrome). ${ }^{5,6}$ Although rare, SCARs are unpredictable and are associated with high morbidity and mortality. ${ }^{6}$ They typically manifest as fever, malaise, cutaneous erythema, progressive blistering, epidermolysis, and mucosal erosions with systemic involvement. ${ }^{6}$ In the majority of cases, a hypersensitivity reaction to drug exposure is the trigger for SCARs, although viral or bacterial infections can also trigger SCARs in the absence of drug exposure. ${ }^{7}$

SCARs are associated with many drugs but do not occur in all patients. ${ }^{8}$ In the EuroSCAR study, a European case-control surveillance of SCARs that was conducted in six countries between 1997 and 2001, and included 379 subjects with SCARs and 1,505 control subjects, allopurinol was the drug most frequently associated with
SCARs (17.4\% cases exposed vs $1.9 \%$ exposed controls; adjusted odds ratio: 18 [95\% confidence interval: 11, 32]), followed by carbamazepine (8.2\% cases exposed) and cotrimoxazole (6.3\% cases exposed). In this study, $3.7 \%$ of the SCAR cases were exposed to lamotrigine. ${ }^{8}$

GSK's lamotrigine is approved for the treatment of epilepsy and bipolar disorder. Skin rash is recognized as a very common side effect of lamotrigine, and serious rashes (including SCARs) have been reported. ${ }^{9}$ Other commonly used antiepileptic drugs, such as carbamazepine, phenobarbital, and phenytoin, are also associated with severe dermatological events such as SJS and TEN. ${ }^{10-13}$ Known risk factors for serious rashes with lamotrigine include the following: starting at a higher-than-recommended initial dose, rapid titration, concurrent valproate administration, a prior history of anticonvulsant-associated rash, and age less than 13 years when the rash develops. ${ }^{14,15}$

Although many studies have been conducted to evaluate the incidence of rash with lamotrigine use, ${ }^{16}$ to date, very few published analyses have evaluated the incidence and causes of fatal SCARs with lamotrigine. This is not entirely surprising considering the rarity of such cases, the known limitations of spontaneous case reports, and the difficulty in estimating the exposure to a medicine in the postmarketing setting. ${ }^{4}$ However, following an increase in the occurrence of fatal SCAR cases in Japan in 2014, this analysis was conducted by GSK to explore the global incidence of fatal SCAR cases following lamotrigine exposure. This permitted a comparison of estimated incidence rates by country, as well as identification of known risk factors that may have contributed to the development of SCAR or the fatal outcome in these cases.

This investigation was performed after additional local risk-mitigation activities were initiated in Japan, which included the issuance of a "Blue Letter" (a "Dear Health Care Provider" letter) in February 2015 in response to the occurrence of fatal SCAR cases in Japan in 2014 and as requested by the Japanese Ministry of Health, Labour and Welfare (MHLW). ${ }^{17}$

\section{Methods}

As part of routine pharmacovigilance, GSK (Brentford, Middlesex, UK) reviews certain individual case safety reports (ICSRs) and aggregate safety data from its global clinical safety database of AE reports. Reports of AEs after drug treatment may be received by GSK through spontaneous reporting by health care professionals (HCPs), regulatory authorities, and members of the public. Other sources of information include the published literature, postmarketing 
surveillance reports, and unblinded clinical trial AE reports. All AEs are coded using the International Conference on Harmonisation (ICH) Medical Dictionary for Regulatory Activities (MedDRA) to the level of preferred term (PT). ${ }^{18}$ For cases in which important clinical details are lacking, attempts are made to contact the source for additional information. Standardized MedDRA queries (SMQs) are created by a working group of the Council for International Organizations of Medical Sciences, in liaison with ICH. ${ }^{19}$ SMQs are designed to ensure consistency of searches by different users in different databases and are therefore widely accepted by regulatory authorities. ${ }^{19,20}$ Narrow SMQs are those that are highly likely to represent the medical condition of interest. ${ }^{20}$ GSK, uses SMQs as its first search strategy for interrogating its global clinical safety database, whenever an appropriate SMQ is developed.

On 29 January 2015, a comprehensive search of the GSK global safety database was conducted, including all cumulative spontaneous AE reports, postmarketing surveillance reports, and unblinded clinical trial serious AE reports (attributable and nonattributable) with lamotrigine, from launch in 1991 until 26 January 2015, the data lock point. These data were accessed via GSK's safety database and therefore are not freely available. For the analysis of global SCAR incidence, worldwide reports (including Japan) containing the narrow SMQ of SCAR (Table 1), fatal or nonfatal, were retrieved. For the analysis of fatal cases in Japan, only those Japanese cases containing the narrow SMQ of SCAR and a fatal outcome were retrieved and included in this analysis. For each country reporting two or more fatal cases, additional data (including total number of lamotrigine cases, total number of SCAR cases, total number of fatal SCAR cases, date of product launch, and cumulative exposure) were retrieved for comparison with the data from other countries.

Cumulative exposure was calculated using sales volume data from Intercontinental Medical Statistics, which has data from 1 January 1995. Exposure estimates were calculated in patient-years using an algorithm that assumes an average

Table I MedDRA PTs in the narrow SMQ for SCARs Acute generalized exanthematous pustulosis, cutaneous vasculitis, dermatitis bullous, exfoliative dermatitis, generalized exfoliative dermatitis, drug reaction with eosinophilia and systemic symptoms, epidermal necrosis, erythema multiforme, exfoliative rash, oculomucocutaneous syndrome, skin necrosis, Stevens-Johnson syndrome, toxic epidermal necrolysis, toxic skin eruption

Abbreviations: MedDRA, Medical Dictionary for Regulatory Activities; PT, preferred term; SCAR, severe cutaneous adverse reaction; SMQ, standardized MedDRA query. global lamotrigine daily dose of $300 \mathrm{mg}$. This algorithm is used to provide the exposure estimates for the production of periodic safety reports, although the average daily dose for individual countries may vary depending on a number of factors (eg, the severity of the disease, concomitant medications, locally approved doses, and indications). Nevertheless, the authors chose to retain the global average daily dose that is globally accepted by regulatory agencies for periodic safety reports, rather than introducing new bias by nominally choosing different values, for different countries. This enabled the calculation of a crude reporting rate and performance of cross-country comparisons, including the proportion of SCAR cases with a fatal outcome. The crude reporting rate for fatal SCAR was calculated for each country based on the number of fatal SCAR cases divided by the cumulative patient exposure in that country.

For the analysis of fatal cases, retrieved reports were carefully reviewed for potential explanations for the fatal outcomes (eg, noncompliance with the prescribing information [PI], concomitant medications, comorbidities, or other risk factors) and for the overall quality of the reported data. Ethics approval was not required as this is a pharmacovigilance evaluation of anonymized spontaneous $\mathrm{AE}$ reports without access to direct patient information.

\section{Results}

Worldwide, the estimated cumulative exposure of lamotrigine was $>8.4$ million patient-years, and there were 54,513 spontaneous AE reports for lamotrigine, of which 3,454 $(6.3 \%)$ reported a SCAR. Of these, $122(3.5 \%)$ had a fatal outcome, equating to a crude rate of 0.01 fatal SCAR cases per 1,000 patient-years. Reporting rates for fatal SCAR cases varied according to country, with the lowest rates reported in the USA, UK, and Spain ( $<0.01$ per 1,000 patient-years), and the highest rate reported in Japan $(0.12$ per 1,000 patientyears; Table 2).

In total, 742 SCAR cases were reported in Japan with lamotrigine, of which 17 (2.3\%) had a fatal outcome. Of these fatal cases, 12 (71\%) showed evidence of noncompliance with the recommended dosing regimen/titration schedule, including a higher initial dose and/or faster escalation schedule than that detailed in the PI (Table 3). Reported starting doses in the 12 cases ranged from $50 \mathrm{mg} / \mathrm{d}$ to $125 \mathrm{mg} / \mathrm{d}$. In 11 cases $(65 \%)$, there was evidence of a delay (ranging from 2 days to 1 week) in the diagnosis and/or management of the early signs of hypersensitivity, including failure to promptly withdraw lamotrigine therapy for the patient. In seven of the cases (41\%), patients had preexisting medical conditions that could have contributed or been potentially responsible for the 


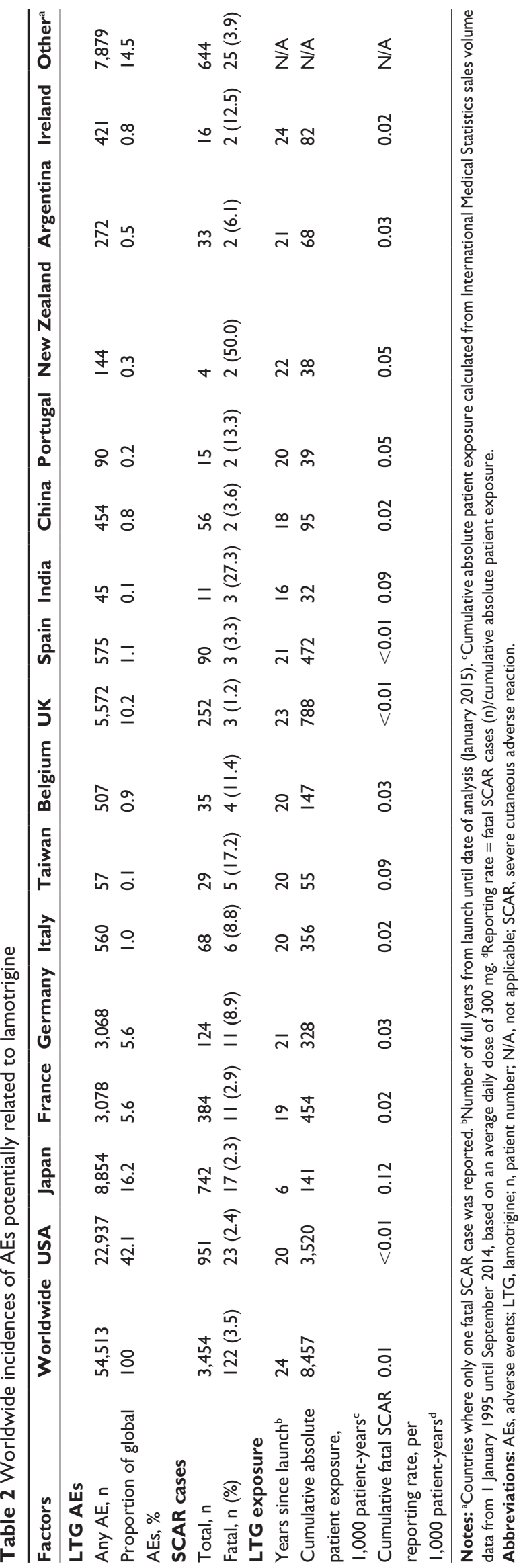

Table 3 Case review findings for fatal SCAR cases, including nonattributable cases

\begin{tabular}{|c|c|c|}
\hline \multirow[t]{2}{*}{ Case review findings } & \multicolumn{2}{|c|}{ Cases, n (\%) ${ }^{a}$} \\
\hline & $\begin{array}{l}\text { Rest of } \\
\text { the world }\end{array}$ & Japan \\
\hline All fatal SCAR cases & $105(100)$ & $17(100)$ \\
\hline \multicolumn{3}{|l|}{ Case review findings } \\
\hline $\begin{array}{l}\text { Possible/definite noncompliance } \\
\text { with dosing regimen }\end{array}$ & $19(18)$ & $12(7 \mid)$ \\
\hline $\begin{array}{l}\text { Possible/definite delay in diagnosis } \\
\text { or management }\end{array}$ & $12(11)$ & II (65) \\
\hline $\begin{array}{l}\text { Possible/definite preexisting medical } \\
\text { condition }\end{array}$ & 0 & $7(4 I)$ \\
\hline History of hypersensitivity to AED & $7(7)$ & $4(24)$ \\
\hline $\begin{array}{l}\text { Use in unauthorized indication, } \\
\text { medication error, abuse, or overdose }\end{array}$ & $6(6)$ & 0 \\
\hline Poorly documented ${ }^{c}$ & $57(54)$ & $\mathrm{I}(6)$ \\
\hline
\end{tabular}

Notes: aSome cases fell into more than one category. ${ }^{\mathrm{b} E x c l u d i n g ~ J a p a n . ~}{ }^{\mathrm{C} T h e r e ~ w a s}$ insufficient information in the case reports to categorize these cases.

Abbreviations: AED, anti-epileptic drug; n, patient number; SCAR, severe cutaneous adverse reaction.

fatal outcome (including cancer, cardiorespiratory arrest, and pneumonia). Only one case (6\%) was considered to be too poorly documented to allow for a meaningful assessment.

By comparison, 105 fatal SCAR cases with lamotrigine were reported from countries other than Japan. The proportion of these cases with evidence of noncompliance with the PI was lower than that for Japan (Table 3). In total, $18 \%$ of the fatal cases reviewed showed evidence of noncompliance with the recommended dosing regimen/titration schedule, and $11 \%$ showed evidence of a delay in the diagnosis and/or management of the early signs of hypersensitivity. However, a much higher percentage of poorly documented cases was seen globally compared with Japan. The majority (54\%) of reports associated with these cases lacked sufficient relevant medical information to allow any meaningful assessments regarding compliance with the PI, compared with the single case $(6 \%)$ from Japan.

\section{Discussion}

This analysis provides an insight into the challenges of managing pharmacovigilance globally. The limitations of spontaneous case reports are always taken into account when signal detection activities are conducted by central safety departments. ${ }^{2}$ Lamotrigine presents an interesting case study because it has been launched globally over a prolonged period. Lamotrigine was first approved in November 1990, with many countries launching the initial and future indications throughout the 1990s and early years from 2000 . In contrast, lamotrigine was not licensed in Japan until 2008 for 
epilepsy and, shortly afterward, in 2011 for bipolar disorder. This meant that the safety profile was already well characterized and described within the PI. ${ }^{9}$ It also meant that the global clinical safety database was extensively populated with AEs reported with lamotrigine, including those that are only rarely reported, such as SCARs. This presents a challenge for global teams who are seeking to identify small changes in the trend of safety data against the backdrop of an established database. It therefore emphasizes the need for collaboration with local safety departments and local regulatory agencies, as they receive new case reports within their country.

Pharmacovigilance departments can also introduce differences in analyses of safety data by using different search strategies during the interrogation of safety data. As reported in this global analysis, 17 cases were identified utilizing a search strategy that used a narrow MedDRA SMQ, retrieving attributable and nonattributable cases. In contrast, the local analysis that was reported in the Japanese Blue Letter identified and reported 16 cases of SCAR in Japan with a fatal outcome. ${ }^{17}$ These 16 cases were identified using different search criteria (MedDRA System Organ Class), and nonattributable cases were excluded. Further investigation of these 16 cases identified that one of them did not involve lamotrigine, making the final number of cases 15 . The broader SMQ-based search strategy used in the global analysis reported here permitted comparison across all countries in which lamotrigine is marketed by GSK and where cases of SCAR have been reported.

Following a detailed review of all the fatal SCAR case reports from Japan, the majority of cases showed evidence of nonadherence to the guidance in the PI regarding dose regimen and/or treatment discontinuation, both of which are known risk factors for the occurrence of SCARs with lamotrigine. ${ }^{13,21}$ In contrast, nonadherence to the dosing regimen/treatment discontinuation guidance was less common among the global cases, as seen in spontaneous reports. The opportunity for further comparison between countries is limited by the low incidence of reported fatal SCARs.

Many potential factors may have contributed to the country-based differences observed in this analysis. Almost all cases of life-threatening rashes reported with lamotrigine have occurred within 2-8 weeks of treatment initiation. ${ }^{21}$ Given that lamotrigine was launched later in Japan compared with the launch in other countries, the proportion of patients under long-term treatment is less. Therefore, patients in the higher-risk early phase of therapy will account for a greater proportion of the total patient exposure in Japan. The reduced time between the launch of the two indications in Japan also provided less time for prescribers to become familiar with the dosing requirements in the epilepsy indication before the bipolar disorder indication was launched. A limitation of this analysis is that these differences were not adjusted for.

When discussing limitations, it is also noteworthy that a far greater proportion of the global cases lacked vital medical information, which made them difficult to assess. Of course, this is a well-known issue with spontaneous case reports. ${ }^{4}$ However, the contrast in the quality of information in spontaneous reports received from Japan, compared with those from the remaining regions of the world, may be an important factor in the apparent lower rate of noncompliance seen globally, compared with Japan. These data also suggest that there may be a higher rate of spontaneous safety case reporting from Japan compared with that in other countries, in addition to this higher quality of reporting. These differences introduce elements of both reporting and recall bias into the analysis. Additionally, the estimated total number of patients receiving lamotrigine is based on sales volume data and an assumed daily dose. These data are cumulative and do not provide sufficient detail to differentiate between new or chronic treatment use. Furthermore, the daily dose is likely to be lower in Japan than in other countries due to regional differences in the approved maximum daily dose. ${ }^{21,22}$ Therefore, the use of exposures estimated in this way introduces further potential bias. The analysis is further limited by reporting bias due to its reliance on spontaneous reporting, which tends to underestimate the true incidence of AEs and which can vary widely between countries according to factors such as the quality of the information provided, awareness and ease of use of local AE reporting systems, and clinicians' experience of the drug in question. ${ }^{2}$ Other limitations of this analysis include the dynamic nature of a safety database due to the ongoing addition of new cases and updates of existing cases with new information, the lack of formal statistical analyses, and the lack of correction for other potential confounding factors, such as concomitant medications, and the patients' underlying medical conditions.

Despite these limitations, GSK considered that there was an increase in the reporting rate of SCAR cases with a fatal outcome in Japan and therefore enhanced the educational program that supported HCPs in the correct use of lamotrigine. This experience with lamotrigine also highlights a potential risk of having a prolonged launch schedule for medicines, particularly those with complicated dosing regimens. For countries that launch later, there is the obvious benefit that the safety profile is well understood, having been used extensively in other regions. However, there is also the risk 
that small increases in the reporting of AEs are not obvious among the total data set, as presented in this case study. This emphasizes the need for both robust local pharmacovigilance that is focused on reports from individual countries as well as close collaboration with central safety departments. This is needed to help place local findings in the context of the global picture and determine when local action is needed.

In response to the occurrence of fatal SCAR cases in Japan in 2014, MHLW asked GSK Japan to distribute a Blue Letter in February 2015. ${ }^{17}$ In addition, several other risk-mitigation activities, including a significant educational program, were initiated. These included adding a warning to the front page of the Japanese PI, ${ }^{22}$ online seminars on lamotrigine safety, educational lectures in local medical conferences, and reinforcement of the need to comply with the PI regarding the correct usage of the drug in ongoing communications with HCPs. The Blue Letter and the additional ongoing risk-mitigation activities conducted by GSK Japan have demonstrated that additional targeted education of prescribers can have a highly positive impact on the safety of patients, leading to a decrease in spontaneous reports of serious skin rashes due to the noncompliant use of lamotrigine.

\section{Conclusion}

This analysis reinforces the importance of pharmacovigilance for well-established products with staggered global launch dates. When launching an established drug into a new region, although there may be substantial clinical experience globally and a well-defined safety profile, it is vital to ensure that prescribers and patients in the new region are fully informed regarding potential adverse effects of the medicine. This can present a major challenge to central safety departments as they establish a global approach to pharmacovigilance. Strong collaboration with local operating companies are essential in order to facilitate the early detection of new safety signals and the rapid development and implementation of local risk-mitigation activities.

This report also demonstrates the importance of using a medicine in accordance with the PI. In order to reduce the risk of SCARs with lamotrigine, it is essential to start at the correct dose and titrate in line with the PI. It is also important to ensure that both patients and HCPs are aware of potential interactions with concomitant medications such as valproate, that there is a possibility that rash may occur, and that lamotrigine should be withdrawn immediately in this instance. The local risk-mitigation activities have demonstrated a positive response to the additional targeted education of prescribers in Japan and have resulted in a reduction in reports of fatal cases of SCAR. This is an early indication of the effectiveness of these measures; however, it is important that vigilance is maintained long term.

\section{Acknowledgments}

This study was funded by GSK. GSK's role included study concept and design, analysis of data, development, and funding of the manuscript. Medical writing and editorial assistance were provided by Paul O'Regan, PhD, of Fishawack Indicia Ltd, UK, also funded by GSK. The MedDRA ${ }^{\circledR}$ trademark is owned by International Federation of Pharmaceutical Manufacturers and Associations on behalf of ICH.

\section{Author contributions}

$\mathrm{HS}, \mathrm{NB}$, and $\mathrm{MC}$ were involved in the study conception and design; HS was involved in acquisition of data; HS, NB, PY, $\mathrm{AK}, \mathrm{GD}$, and $\mathrm{MC}$ contributed to the analysis and interpretation of data; all authors took part in drafting the article or revising it critically for important intellectual content; gave final approval of the version to be published; and agree to be accountable for all aspects of the work.

\section{Disclosure}

All authors are current or former employees of GSK and hold stocks/shares in GSK. The authors report no other conflicts of interest in this work.

\section{References}

1. Dal Pan GJ. Ongoing challenges in pharmacovigilance. Drug Safety. 2014;37(1):1-8.

2. Hazell L, Shakir SA. Under-reporting of adverse drug reactions: a systematic review. Drug Safety. 2006;29(5):385-396.

3. US Food and Drug Administration. Good Pharmacovigilance Practices and Pharmacoepidemiologic Assessment. 2005. Available from: https:// www.fda.gov/downloads/regulatoryinformation/guidances/ucm126834. pdf. Accessed April 2017.

4. Dal Pan GJ, Lindquist M, Gelperin K. Postmarketing Spontaneous Pharmacovigilance Reporting Systems. In Strom BL, Kimmel SE, Hennessy S, editors. Textbook of Pharmacoepidemiology. Chichester: John Wiley \& Sons Ltd; 2013:137-158.

5. Dao RL, Su SC, Chung WH. Recent advances of pharmacogenomics in severe cutaneous adverse reactions: immune and nonimmune mechanisms. Asia Pac Allergy. 2015;5(2):59-67.

6. Struck MF, Hilbert P, Mockenhaupt M, Reichelt B, Steen M. Severe cutaneous adverse reactions: emergency approach to non-burn epidermolytic syndromes. Intensive Care Med. 2010;36(1):22-32.

7. Harr T, French LE. Toxic epidermal necrolysis and Stevens-Johnson syndrome. Orphanet J Rare Dis. 2010;5:39.

8. Halevy S, Ghislain PD, Mockenhaupt M, et al; EuroSCAR Study Group. Allopurinol is the most common cause of Stevens-Johnson syndrome and toxic epidermal necrolysis in Europe and Israel. J Am Acad Dermatol. 2008;58(1):25-32.

9. Lamictal [webpage on the internet]. Surry: eMC [updated March 20, 2017]. Available from: http://www.medicines.org.uk/emc/medicine/4228. Accessed June 20, 2017. 
10. Phenobarbital Actavis Tablets BP $30 \mathrm{mg}$ [webpage on the Internet]. Surry: eMC [updated December 30, 2013]. Available from: https:// www.medicines.org.uk/emc/medicine/24077. Accessed August, 2016.

11. Phenytoin Sodium Flynn Hard Capsules $25 \mathrm{mg}, 50 \mathrm{mg}, 100 \mathrm{mg}$ and $300 \mathrm{mg}$ [webpage on the internet]. Surry: eMC [updated November 9, 2016]. Available from: https://www.medicines.org.uk/ emc/medicine/28811. Accessed June 20, 2017.

12. Levi N, Bastuji-Garin S, Mockenhaupt M, et al. Medications as risk factors of Stevens-Johnson syndrome and toxic epidermal necrolysis in children: a pooled analysis. Pediatrics. 2009;123(2):e297-e304.

13. Mockenhaupt M, Viboud C, Dunant A, et al. Stevens-Johnson syndrome and toxic epidermal necrolysis: assessment of medication risks with emphasis on recently marketed drugs. The EuroSCAR-study. J Invest Dermatol. 2008;128(1):35-44.

14. Guberman AH, Besag FM, Brodie MJ, et al. Lamotrigine-associated rash: risk/benefit considerations in adults and children. Epilepsia. 1999;40(7):985-991.

15. Wang XQ, Lv B, Wang HF, et al. Lamotrigine-induced severe cutaneous adverse reaction: update data from 1999-2014. J Clin Neurosci. 2015;22(6):1005-1011.

16. Seo HJ, Chiesa A, Lee SJ, et al. Safety and tolerability of lamotrigine: results from 12 placebo-controlled clinical trials and clinical implications. Clin Neuropharmacol. 2011;34(1):39-47.
17. Pharmaceuticals and Medical Devices Agency. Dear Healthcare Professionals Letter of Rapid Safety Communication (Blue Letter), Serious Skin Disorders Suggestively Caused by Lamictal (Lamotrigine) Tablets. 2015. Available from: http://www.pmda.go.jp/files/000198527.pdf. Accessed April 2016.

18. meddra.org [homepage on the Internet]. Medical Dictionary for Regulatory Activities. Available from: http://www.meddra.org/. Accessed June 18, 2017.

19. Mozzicato P. Standardised MedDRA queries: their role in signal detection. Drug Safety. 2007;30(7):617-619.

20. Medical Dictionary for Regulatory Activities [webpage on the Internet]. Standardised MedDRA Queries. 2016. Available from: http://www. meddra.org/standardised-meddra-queries. Accessed December 2016.

21. GlaxoSmithKline. Lamictal US Prescribing Information. 2016. Available at: https://www.gsksource.com/pharma/content/dam/GlaxoSmithKline/ US/en/Prescribing_Information/Lamictal/pdf/LAMICTAL-PI-MG.PDF. Accessed June 20, 2017.

22. Pharmaceuticals and Medical Devices Agency. Pharmaceuticals and Medical Devices Safety Information, No. 321 March 2015. 2015. Available from: https://www.pmda.go.jp/files/000205168.pdf. Accessed April 2016.
Therapeutics and Clinical Risk Management

\section{Publish your work in this journal}

Therapeutics and Clinical Risk Management is an international, peerreviewed journal of clinical therapeutics and risk management, focusing on concise rapid reporting of clinical studies in all therapeutic areas, outcomes, safety, and programs for the effective, safe, and sustained use of medicines. This journal is indexed on PubMed Central, CAS,

\section{Dovepress}

EMBase, Scopus and the Elsevier Bibliographic databases. The manuscript management system is completely online and includes a very quick and fair peer-review system, which is all easy to use. Visit http://www.dovepress.com/testimonials.php to read real quotes from published authors.

Submit your manuscript here: http://www.dovepress.com/therapeutics-and-clinical-risk-management-journal 